\title{
Comparison of effects of glass fibre and glass powder on guinea-pig lungs
}

\author{
SUSAN K. BOTHAM and P. F. HOLT \\ Department of Chemistry, University of Reading
}

\begin{abstract}
Botham, Susan K., and Holt, P. F. (1973). British Journal of Industrial Medicine, 30, 232-236. Comparison of effects of glass fibre and glass powder on guinea-pig lungs. Following 24 hours inhalation by guinea-pigs of powdered glass dust, the pulmonary effects over the succeeding month differed from those previously observed to follow inhalation of glass fibre in that (1) fewer erythrocytes escaped from the capillaries, (2) very few giant cells were produced, (3) erythrocytes and intracellular glass particles were cleared more readily because junctions between respiratory and terminal bronchioles were not blocked by giant cells, (4) intracellular granules containing Perls-positive material did not appreciably increase in number or intensity of staining during the month, and (5) particles were not coated with Perls-positive material during the time that pseudo-asbestos bodies would be formed from glass fibres. The difference between the effects of chemically similar glass powder and fibre during a month in a guinea-pig lung is considered to be due to the morphology of the inhaled particle.
\end{abstract}

Beck, Holt, and Manojlović (1972) established that chrysotile asbestos and glass fibre caused similar changes in macrophage cultures, producing an increase in cell membrane permeability which is demonstrable by the increase in lactic dehydrogenase activity in the supernatant fluid. The metabolism, measured by lactate production, is not reduced as it is when quartz is phagocytosed. Glass powder, chemically similar to the fibres, behaved like an inert dust, producing only a small increase in lactate dehydrogenase activity and an increase in lactate production as a result of higher metabolism due to phagocytosis. They considered that the slow and sometimes incomplete process of ingestion of long fibres resulted in an increase in the permeability of the macrophage membrane and allowed the leakage of cytoplasmic constituents from the cell.

The changes in the guinea-pig lung that follow the inhalation of asbestos (Botham and Holt, 1968) and glass fibre (Botham and Holt, 1971a) have been described. One of the early effects resulting from the inhalation of any of these fibrous dusts is the diapedesis of erythrocytes through the walls of capillaries near to the fibres (Botham and Holt,
1968, 1971a and b, 1973) and it has been suggested (Beck, Holt, and Nasrallah, 1971) that a substance affecting capillary permeability may leak from the macrophage during the process of phagocytosis. In this paper the effects of glass powder on the lungs of guinea-pigs is compared with the effects of glass fibre of the same composition.

\section{Materials and methods}

Glass spheres (supplied by Johns-Manville Fiber Glass Inc.) were fused into a mass and placed with other spheres in a wooden tumbler box lined with rubber. When the box was rotated the mutual grinding action of the glass lump and spheres produced a fine glass powder, which was then drawn in a current of air into a dusting tunnel. A sample of the powdered glass was taken on a microscope slide from the dusting tunnel during the subsequent inhalation experiment. The particles were found to be irregular in outline, most having a maximum dimension below $10 \mu \mathrm{m}$, but occasionally larger particles of up to $100 \mu \mathrm{m}$ were found. The particles did not give a positive reaction when tested with Perls reagent.

Ten albino male guinea-pigs were exposed in a dusting tunnel to an atmosphere with a high concentration of these glass particles. One animal was removed and killed 
after 3 hours dusting, but the rest were exposed for a further 21 hours. Other animals were killed after 1, 2, 3, $4,5,7,14,21$, and 28 days after dusting began. One control animal was killed at the beginning and a second at the end of the experiment.

The lungs were fixed in buffered neutral $4 \%$ formol saline and paraffin-wax sections were prepared and stained with Perls' reagent alone or with Perls' reagent and Harris's haematoxylin and eosin (PHE). Photomicrographs of the sections were taken with a Leitz Ortholux.

\section{Results}

The glass particles were usually seen in dense groups, in which each particle had a maximum diameter of $1 \mu \mathrm{m}$. Occasionally large isolated particles were found; the greatest dimension across such a particle was $45 \mu \mathrm{m}$, but the majority measured less than $15 \mu \mathrm{m}$.

Groups of fine extracellular particles were seen in the mucus within the lumina of the larger bronchioles of the two guinea-pigs killed directly after dusting. From four days after the beginning of dusting, the majority of glass particles found in bronchiolar debris were intracellular (Fig. 1a, b), but in animals killed later there were still groups of extracellular glass particles in some bronchioles.

Macrophages containing many fine glass particles were found in the bronchiolar debris of all guineapigs. There were very few giant cells. The first example of a giant cell was found in the guinea-pig killed four days after the beginning of dusting, but none was found in the three animals killed in the final three weeks of the experiment. In all guineapigs, even in the animal killed after only 3 hours' exposure, the bronchiolar debris contained red cells (Fig. 1a, b). The number of red cells decreased with increasing time after exposure, although a few red cells were still found in some of the large bronchioles of the final animal. First extracellular, and then intracellular, glass particles and red cells were rapidly cleared in the bronchiolar debris, so that in the final three animals some of the terminal bronchioles were clear.

A few glass particles were found free in respiratory bronchioles in the guinea-pig killed immediately after 3 hours of dusting. Later, practically all glass particles were intracellular, either ingested by phagocytes in the walls of these bronchioles or in cells which had become detached.These cells were generally macrophages with vacuolated cytoplasm: again giant cells were very rare. Occasional red cells and eosinophils were found free in some of the respiratory bronchioles. These red cells were less numerous than those seen in the respiratory bronchioles of guinea-pigs killed within a week after glass fibre inhalation (Botham and Holt, 1971a).

Isolated small extracellular glass particles were occasionally found in the alveoli, even in the animal killed one month after exposure to the dust. The largest of these particles had a maximum dimension of $15 \mu \mathrm{m}$. The first macrophage containing powdered glass particles and lying free in an alveolus was found in the guinea-pig killed two days after the beginning of dusting. Further examples occurred in other animals killed up to two weeks after this. In the four animals killed from seven days to a month after dusting, however, groups of macrophages with vacuolated cytoplasm and containing numbers of fine glass particles were found to fill some alveoli completely. It was seldom that macrophages containing the powdered glass particles produced giant cells. Macrophages containing fine glass particles were found in the interalveolar septa of all the guinea-pigs killed after a 24 hour dusting period but giant cells were rarely found. In the animal killed a month after dusting, one instance was found of glass particles lying freely in an alveolus close to a macrophage still packed with particles and forming part of an interalveolar septum.

Perls-positive material was always found to be intracellular and usually appeared as very fine granules which stained pale blue. In all the guineapigs exposed for 24 hours to powdered glass, macrophages containing these Perls-positive granules were found scattered in the interalveolar septa. In the first week these cells were comparatively few, but they became more numerous in the final three animals killed. The granules were stained a uniform pale blue by Perls' technique.

In the first six guinea-pigs killed after inhalation of powdered glass, macrophages that contained Perlspositive granules often did not contain glass particles, and macrophages containing only glass particles were also found. In the guinea-pig killed two days after the beginning of dusting, a few macrophages were seen to contain both glass particles and Perlspositive material; gradually there was an increase in the number of these cells, until in the seventh animal (killed seven days after the beginning of dusting) only a few macrophages that contained glass particles did not additionally contain either Perls-positive cytoplasm or granules. In the final three animals, Perls-positive granules were almost exclusively confined to macrophages that also contained particles of powdered glass (Fig 2a). Some macrophages containing particles and Perls-positive granules had become detached and were first found free in the alveoli (and even in the bronchioles) of the animal killed one day after the 24 hours of exposure to glass particles. A few of these cells were found in alveoli, and in the various generations of bronchioles, of the following four animals killed. In the next two guinea-pigs killed, however, detached phagocytes containing both Perls-positive granules and glass particles were seen only in the larger terminal and cartilaginous bronchioles but were not 


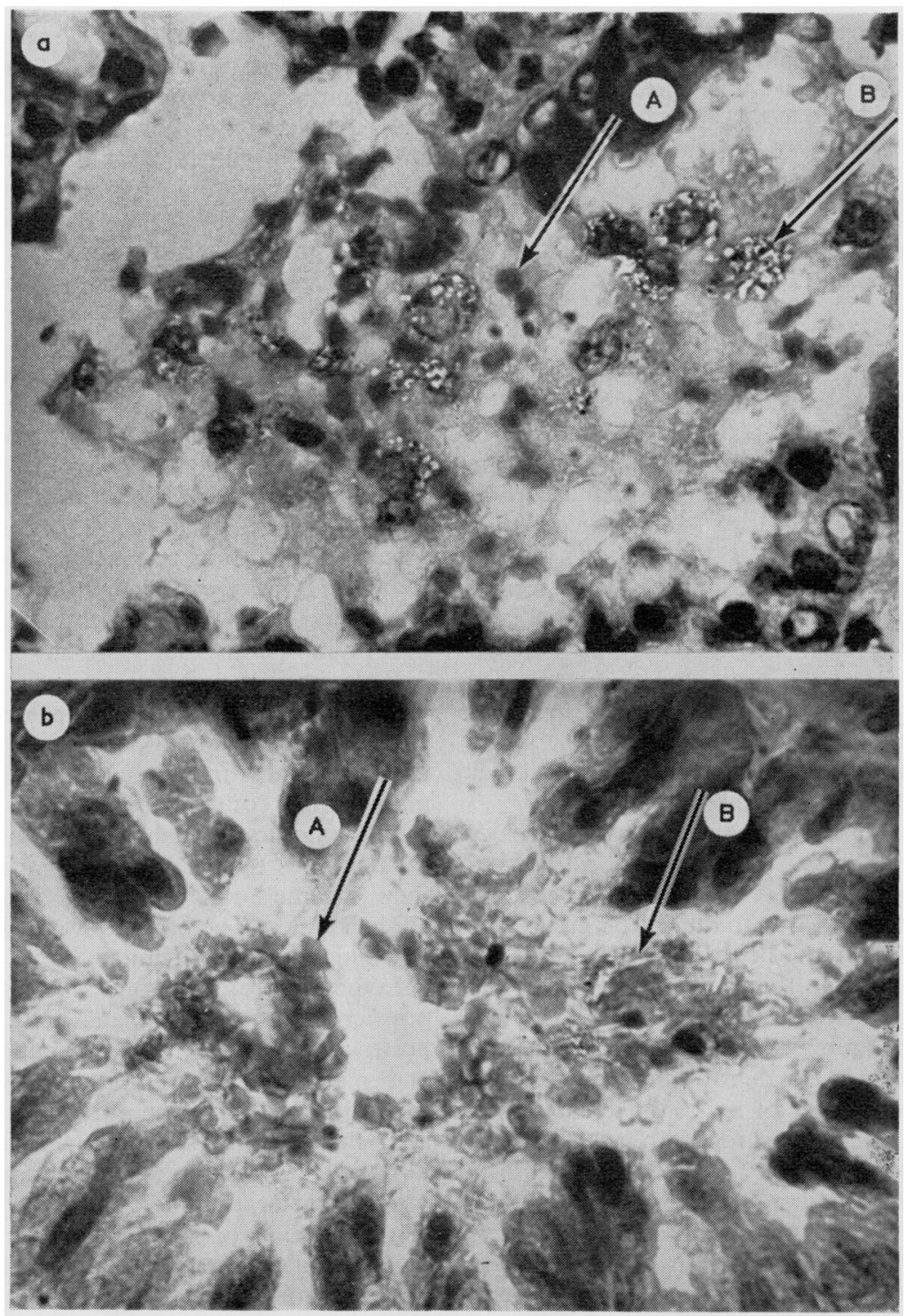

FIG. 1. Comparison between bronchiolar debris following inhalation of glass dusts by guinea-pigs. (a) Debris composed of erythrocytes (arrow A) and particles of glass powder in macrophages (arrow B) in lumen of terminal bronchiole. Guinea-pig received 24 hours exposure to powdered glass dust and was killed four days after beginning of dusting. Perls, Harris haematoxylin, and eosin $\times 1100$. (b) Debris composed of erythrocytes (arrow A) and glass fibres in giant cell (arrow B) in lumen of terminal bronchiole. Guinea-pig received 24 hours exposure to glass fibre dust and was killed four days after beginning of dusting. Perls, Harris haematoxylin, and eosin $\times 1100$.

found in the animal killed at the end of the experiment.

Discrete glass particles and Perls-positive granules were found in many macrophages, but even a month after inhalation no association between glass and
Perls-positive material was found. (It is impossible to prove that the Perls-positive granules contained no minute glass fragments, but the granules in general were very much smaller than most glass particles.) 


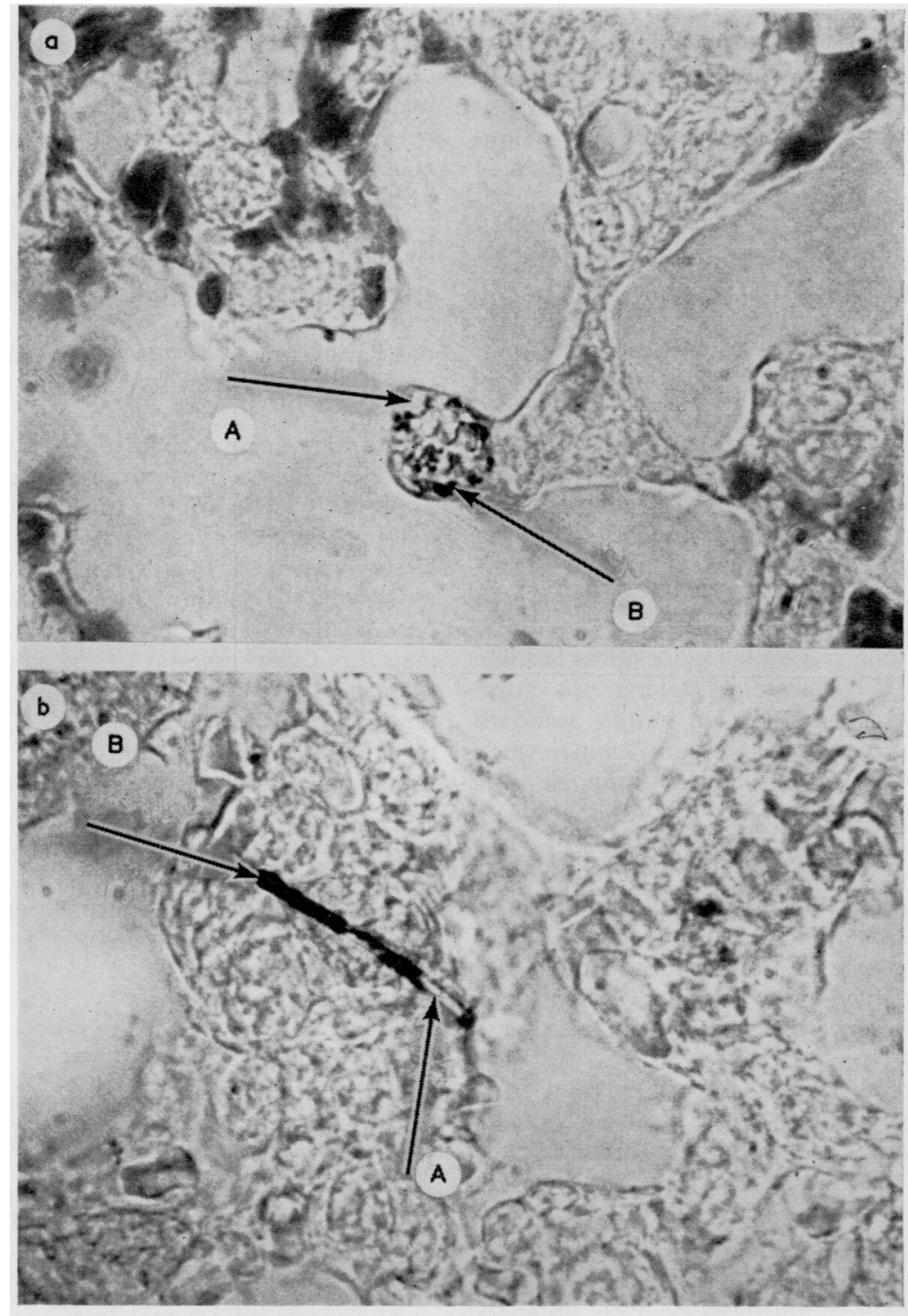

FIG. 2. Comparison between response in alveolar region to inhalation of glass dusts by guinea-pigs. (a) Particles of powdered glass (arrow A) and Perls positive granules (arrow B) in macrophage in interalveolar septum. Guinea-pig received 24 hours exposure to powdered glass dust and was killed 14 days after beginning of dusting. Phase contrast. Perls $\times 2$ 090. (b) Glass fibre (arrow A) with Perlspositive coating (arrow B) in giant cell in interalveolar septum. Guinea-pig received 24 hours exposure to glass fibre dust and was killed 14 days after beginning of dusting. Phase contrast. Perls $\times 2090$.

No Perls-positive material or glass particles were found in the areas of lymphoid tissue in the lungs of any guinea-pig treated with powdered glass.

\section{Comment}

Glass powder affects macrophage cultures in a manner similar to an inert dust such as aluminium oxide. Unlike quartz powder it does not have a toxic effect and it does not cause the leakage of large quantities of lactic dehydrogenase from the cell (Beck et al., 1972). The toxicity of quartz appears to be due to the chemical composition or nature of the surface of the dust. The results of our exper- 
iments, where there was a greater diapedesis following inhalation of glass fibre than of glass powder of the same chemical composition, are in agreement with the tissue culture data where glass fibres increase the permeability of the cell membrane of macrophages to a greater extent than does glass powder.

Following the inhalation of powdered glass by the guinea-pigs, diapedesis of erythrocytes from capillaries into the alveoli occurred and the appearance in macrophages of iron derived from the haemoglobin was indicated by Perls staining reaction. This effect was less extensive, however, than was seen following inhalation of fibrous dusts. Moreover, although appreciable amounts of demonstrable iron were present in sections taken two days after dusting, the amount after one month was much less than in lungs containing an inhaled fibrous dust.

A second difference was in the development of a Perls-positive coating around a fibre in a phagocyte. In a guinea-pig lung some long glass fibres become coated with a ferro-protein material to form pseudo-asbestos bodies (Fig. 2b) within a month after their inhalation but this does not happen when glass powder of the same chemical composition is inhaled (Fig. 2a).

A third difference between the effects of glass fibre and glass powder was seen in the production of giant cells. In the lungs containing glass fibre, two or more macrophages that had ingested different regions of a long fibre had often interdigitated and fused to enclose the fibre completely. Giant cells were rare in the guinea-pigs that had inhaled glass powder.

When giant cells develop after inhalation of a fibrous dust, some become detached and may form plugs blocking distal parts of terminal bronchioles, thus preventing clearance of the alveoli they serve. Following the inhalation of powdered glass, detached macrophages containing particles of glass and Perlspositive material were found in the alveoli and bronchioles where they appeared as loose plugs of debris after seven days, but after 14 and 21 days such phagocytes were found only in the larger terminal and cartilaginous bronchioles. After 28 days even these air spaces have been cleared.

It appears that fibrous material, because of its special morphology, produces a particular type of reaction in the alveoli and it is reasonable to deduce that the pathological effects of asbestos are also due to the morphology of the fibres. It must then be supposed that glass or asbestos fibres retained in the lung will produce the same kind of pathologicaleffect.

The normal method for clearance of those fibres that are not removed in the bronchiolar debris is coating to form an asbestos body or pseudoasbestos body, fragmentation of this structure, and removal of debris by macrophages (Botham and
Holt, 1968, 1971a). The morphology of a fibrous dust determines its ingestion time, leakage of material from the macrophage, increased capillary permeability, subsequent diapedesis, and the appearance of Perls-positive granules. When a fibre has been coated the time elapsing before the asbestos or pseudo-asbestos body fragments is related to the chemical composition of the enclosed fibre. It has been shown (Botham and Holt, 1971a, b) that glass and chrysotile fibres are coated and fragmented more rapidly than are amosite and crocidolite, and that the last two can thus exert a pathogenic effect over a longer period.

It is only during the last few years that glass fibre of respirable dimensions has assumed industrial importance. The widely used grades usually have a fibre diameter exceeding $10 \mu \mathrm{m}$ and fibres are and likely to reach the alveoli. Indeed, Gross, Tuma, and de Treville (1971) found no significant difference unbetween the average number of fibres in the lungs of glass fibre workers and of the normal population. Stanton and Wrench (1972) produced mesotheliomata with glass fibres of $0.06-3 \mu \mathrm{m}$ diameter, as well as with amosite, chrysotile, and crocidolite, following implantation of these materials against the pleura of rats and concluded that the carcinogenicity of these materials is primarily related to structure rather than to physicochemical properties. Our experiment has shown that different responses occur when the shape of an inhaled dust but not its chemical composition is changed.

\section{References}

Beck, E. G., Holt, P. F., and Manojlović, N. (1972). Comparison of effects on macrophage cultures of glass fibre, glass powder, and chrysotile asbestos. British Journal of Industrial Medicine, 29, 280-286.

, - - and Nasrallah, E. T. (1971). Effects of chrysotile and acid-treated chrysotile on macrophage cultures. British Journal of Industrial Medicine, 28, 179-185.

Botham, S. K., and Holt, P. F. (1968). The mechanism of formation of asbestos bodies. Journal of Pathology and Bacteriology, 96, 443-453.

, (1971a). The development of glass-fibre bodies in the lungs of guinea-pigs. Journal of Pathology, 103, 149156.

- - (1971b). Development of asbestos bodies on amosite, chrysotile and crocidolite fibres in guinea-pig lungs. Journal of Pathology, 105, 159-167.

- (1972). Asbestos-body formation in the lungs of rats and guinea-pigs after inhalation of anthophyllite. Journal of Pathology, 107, 245.

Gross, P., Tuma, J., and de Treville, R. T. P. (1971). Lungs of workers exposed to fiber glass. Archives of Environmental Health, 23, 67-76.

Stanton, M. F., and Wrench, C. (1972). Mechanisms of mesothelioma induction with asbestos and fibrous glass. Journal of the National Cancer Institute, 48, 797-821.

Received for publication November 14, 1972.

Accepted for publication March 29, 1973. 